\title{
Eigenvalues for Finsler $p$-Laplacian with zero Dirichlet boundary condition
}

\author{
Maria Fărcăşeanu
}

\begin{abstract}
In this paper we analyze the problem $-Q_{p} u(x)=\lambda u(x)$ when $x \in \Omega$ with $u(x)=0$ when $x \in \partial \Omega$, where $\Omega \subset \mathbb{R}^{N}$ is a bounded domain, $Q_{p}$ stands for Finsler p-Laplacian and $p \in\left(\frac{2 N}{N+2}, \infty\right) \backslash\{2\}$ is a given real number. Using adequate variational methods we show that the set of eigenvalues of this problem is the interval $(0, \infty)$.
\end{abstract}

\section{Introduction and main result}

The goal of this paper is to investigate the eigenvalue problem

$$
\begin{cases}-Q_{p} u(x)=\lambda u(x), & \text { for } x \in \Omega \\ u(x)=0, & \text { for } x \in \partial \Omega,\end{cases}
$$

where $\Omega \subset \mathbb{R}^{N}(N \geq 3)$ is a bounded domain with smooth boundary, $\lambda$ is a positive real number and $p \in\left(\frac{2 N}{N+2}, \infty\right) \backslash\{2\}$. We recall that

$$
Q_{p} u:=\sum_{i=1}^{N} \frac{\partial}{\partial x_{i}}\left(H(\nabla u)^{p-1} H_{x_{i}}(\nabla u)\right)
$$

denotes the Finsler $p$-Laplacian, where $H$ is a Finsler norm, i.e. $H: \mathbb{R}^{N} \rightarrow$ $[0, \infty)$ is a convex function of class $C^{2}\left(\mathbb{R}^{N} \backslash\{0\}\right)$ even and homogeneous of

Key Words: eigenvalue problem; $p$-Laplacian; weak solution.

2010 Mathematics Subject Classification: Primary 35J60; Secondary 35J92; 35J20.

Received: April, 2014.

Revised: May, 2014.

Accepted: June, 2014. 
degree 1 (we refer to a paper by Ferone and Kawohl [4] for the properties of Finsler norms and for some examples). For $H(x)=|x|$ (here $|\cdot|$ denotes the euclidian norm in $\left.\mathbb{R}^{N}\right)$, we have $Q_{p} u=\Delta_{p} u:=\sum_{i=1}^{N} \frac{\partial}{\partial x_{i}}\left(|\nabla u|^{p-2} \nabla u\right)$, where $\Delta_{p}$ denotes the usual $p$-Laplacian. Note that if $H(x)=|x|$ and $p=2$ it is well-known that problem (1) possesses an unbounded sequence of positive eigenvalues (see, e.g. Brezis [2, Theorem IX.31] or [3])

$$
0<\lambda_{1}^{D}<\lambda_{2}^{D} \leq \ldots \leq \lambda_{n}^{D} \leq \ldots
$$

Consequently, in this case we deduce that the set of eigenvalues of problem (1) is discrete. Moreover, each eigenfunction corresponding to any eigenvalue $\lambda_{k}^{D}$ with $k \geq 2$ changes sign in $\Omega$.

Definition 1. We say that $\lambda \in \mathbb{R}$ is an eigenvalue of problem (1), if there exists $u \in W_{0}^{1, p}(\Omega) \backslash\{0\}$ such that

$$
\int_{\Omega}(H(\nabla u))^{p-2} \nabla H(\nabla u) \nabla \varphi d x=\lambda \int_{\Omega} u \varphi d x
$$

for all $\varphi \in W_{0}^{1, p}(\Omega)$. Such a function $u$ will be called an eigenfunction corresponding to the eigenvalue $\lambda$.

Remark. Note that for $N \geq 3$ and $p \in\left(1, \frac{2 N}{N+2}\right)$ we can not define a solution of problem (1) in the sense of Definition 1 since in that case the integral from the right-hand side of relation (2) is not correctly defined. In the context of our approach this situation is related with the fact that the Sobolev embedding of $W_{0}^{1, p}(\Omega)$ in $L^{2}(\Omega)$ fails to hold if $2>N p /(N-p)$ (see, e.g. [2, Theorem IX.16] or [3]).

The main result of this paper is given by the following theorem:

Theorem 1. The set of eigenvalues of problem (1) is given by the interval $(0, \infty)$. Moreover, each eigenvalue possesses a nonnegative corresponding eigenfunction.

Remark. By Theorem 1 we deduce that for each $p \in\left(\frac{2 N}{N+2}, \infty\right) \backslash\{2\}$ the set of eigenvalues of problem (1) is a continuous family. Moreover, for each eigenvalue we can find a corresponding eigenfunction which does not change sign in $\Omega$. This is in sharp contrast with the situation which occurs for $p=2$. Note also that our main result complements some known results by Ôtani [5] where the particular case $H(x)=|x|$ was investigated. 


\section{Proof of the main result}

First, note that if a real number $\lambda$ is an eigenvalue of problem (1) then it is positive. This is a simple remark of the fact that if we replace $\varphi$ with the corresponding eigenfunction $u \neq 0$ in relation (2), we get $\lambda>0$.

Next, our approach will be based on a method described in [8, pp. 118-119] (see also [9]).

We consider the minimization problem

$$
\text { Minimize } \int_{\Omega}(H(\nabla u))^{p} d x
$$

under the assumptions $u \in W_{0}^{1, p}(\Omega)$ and $\|u\|_{L^{2}(\Omega)}=1$.

We will show that the solutions for problem (3) are also solutions of problem (1).

Lemma 1. If there exists $u \geq 0$ a solution of problem (3), then it is a solution of problem (1) with

$$
\lambda:=\inf _{w \in W_{0}^{1, p}(\Omega),\|w\|_{L^{2}(\Omega)}=1} \int_{\Omega}(H(\nabla w))^{p} d x .
$$

Proof. Let $u \in W_{0}^{1, p}(\Omega)$ be a solution of (3), i.e. $\|u\|_{L^{2}(\Omega)}=1$ and $\int_{\Omega}(H(\nabla u))^{p} d x=\lambda$. We fix $\varphi \in W_{0}^{1, p}(\Omega)$ arbitrarily. Define $f: \mathbb{R} \rightarrow \mathbb{R}$ by

$$
f(\epsilon)=\int_{\Omega}(H(\nabla(u+\epsilon \varphi)))^{p} d x-\lambda\left(\int_{\Omega}(u+\epsilon \varphi)^{2} d x\right)^{\frac{p}{2}} .
$$

Clearly, there exists $\delta>0$ such that $u+\epsilon \varphi \not \equiv 0$ for all $\epsilon \in(-\delta, \delta)$. By (4) we deduce that

$$
\lambda=\inf _{w \in W_{0}^{1, p}(\Omega) \backslash\{0\}} \frac{\int_{\Omega}(H(\nabla w))^{p} d x}{\left(\int_{\Omega}|w|^{2} d x\right)^{\frac{p}{2}}} .
$$

Then $f(\epsilon) \geq 0, \forall \epsilon \in \mathbb{R}$ and $f(0)=0$, so $\epsilon=0$ is a global minimum point for $f$. By Fermat's theorem we get that $f^{\prime}(0)=0$. We deduce that

$$
\begin{gathered}
f^{\prime}(\epsilon)=p \int_{\Omega}(H(\nabla(u+\epsilon \varphi)))^{p-2} \nabla H(\nabla(u+\epsilon \varphi)) \nabla \varphi d x \\
-\lambda \frac{p}{2}\left(\int_{\Omega}(u+\epsilon \varphi)^{2} d x\right)^{\frac{p}{2}-1} 2 \int_{\Omega}(u+\epsilon \varphi) \varphi d x .
\end{gathered}
$$


Therefore

$$
0=f^{\prime}(0)=p \int_{\Omega}(H(\nabla u))^{p-2} \nabla H(\nabla u) \nabla \varphi d x-\lambda p \int_{\Omega} u \varphi d x
$$

which proves that $u$ is a solution for problem (1).

Lemma 2. There exists $u \in W_{0}^{1, p}(\Omega) \backslash\{0\}, u \geq 0$ solution of problem (3).

Proof. Let $\left\{u_{n}\right\}_{n} \in W_{0}^{1, p}(\Omega)$ be a minimizing sequence for $\lambda$, that is

$$
\left\|u_{n}\right\|_{L^{2}(\Omega)}=1, \text { for every } n \text { and } \int_{\Omega}\left(H\left(\nabla u_{n}\right)\right)^{p} d x \rightarrow \lambda \text { as } n \rightarrow \infty \text {. }
$$

So, $\left\{u_{n}\right\}_{n}$ is bounded in $W_{0}^{1, p}(\Omega)$, which implies that there exists $u \in W_{0}^{1, p}(\Omega)$ such that $u_{n}$ converges weakly to $u$ in $W_{0}^{1, p}(\Omega)$. Since $H$ is convex the fact that $u_{n}$ converges weakly to $u$ in $W_{0}^{1, p}(\Omega)$ implies

$$
\int_{\Omega}(H(\nabla u))^{p} d x \leq \liminf _{n \rightarrow \infty} \int_{\Omega}\left(H\left(\nabla u_{n}\right)\right)^{p} d x .
$$

As $W_{0}^{1, p}(\Omega)$ is embedded compactly in $L^{2}(\Omega)$ for $p \in\left(\frac{2 N}{N+2}, \infty\right) \backslash\{2\}$, we deduce that $u_{n}$ converges strongly to $u$ in $L^{2}(\Omega)$ and as $\left\|u_{n}\right\|_{L^{2}(\Omega)}=1$, we conclude that $\|u\|_{L^{2}(\Omega)}=1$. From the definition of $\lambda$ we obtain

$$
\lambda=\int_{\Omega}(H(\nabla u))^{p} d x
$$

We can take $|u(x)|$ instead of $u(x)$ and conclude that problem (3) has a solution $u(x) \geq 0$ in $\Omega$.

Theorem 2. Let $\Omega \subset \mathbb{R}^{N}(N \geq 3)$ be a bounded domain with smooth boundary and $p \in\left(\frac{2 N}{N+2}, \infty\right) \backslash\{2\}$. Then, for every $\mu \geq 0$, problem

$$
\begin{cases}-Q_{p} v(x)=\mu v(x), & \text { for } x \in \Omega \\ v(x)=0, & \text { for } x \in \partial \Omega \\ v(x) \geq 0, & \text { for } x \in \Omega\end{cases}
$$

has a nontrivial solution.

Proof. By Lemmas 1 and 2, there exists $u \in W_{0}^{1, p}(\Omega) \backslash\{0\}, u \geq 0$, a solution of problem (1) with

$$
\lambda:=\inf _{w \in W_{0}^{1, p}(\Omega),\|w\|_{L^{2}(\Omega)}=1} \int_{\Omega}(H(\nabla w))^{p} d x
$$


and $\int_{\Omega}(H(\nabla u))^{p-2} \nabla H(\nabla u) \nabla \varphi d x=\lambda \int_{\Omega} u \varphi d x$ for all $\phi \in W_{0}^{1, p}(\Omega)$.

Consider $\mu>0$ fixed. Let $t>0$ such that $t^{p-2}=\frac{\mu}{\lambda}$ and $v:=t u, v \in$ $W_{0}^{1, p}(\Omega) \backslash\{0\}$. Since $H$ is homogeneous of degree 1 (i.e., $H(t x)=|t| H(x)$ for all $t \in \mathbb{R}$ and all $\xi \in \mathbb{R}^{N}$ ) we find that

$$
\begin{gathered}
\int_{\Omega}(H(\nabla v))^{p-2} \nabla H(\nabla v) \nabla \varphi d x=t^{p-1} \int_{\Omega}(H(\nabla u))^{p-2} \nabla H(\nabla u) \nabla \varphi d x \\
=t^{p-1} \lambda \int_{\Omega} u \varphi d x=t^{p-1} \lambda \int_{\Omega} \frac{v}{t} \varphi d x \\
=t^{p-2} \lambda \int_{\Omega} v \varphi d x \\
=\mu \int_{\Omega} v \varphi d x, \forall \varphi \in W_{0}^{1, p}(\Omega),
\end{gathered}
$$

which concludes the proof.

\subsection{An alternative proof of Theorem 1 in the case $p \in(2, \infty)$}

In order to analyze problem $(1)$ when $p \in(2, \infty)$ we can also use the so-called Direct Method in the Calculus of Variations (see [6, Theorem 1.2]).

Theorem 3. Assume $X$ is a reflexive Banach space and let $M \subseteq X$ be a nonempty, weakly closed subset of $X$. Assume $I: M \rightarrow \mathbb{R} \cup\{\infty\}$ is coercive on $M$ with respect to $X$ and weakly lower semi-continuous on $M$ with respect to $X$. Then, $I$ is bounded from below on $M$ and attains its minimum in $M$.

In order to apply Theorem 3, we define for each arbitrary but fixed $\lambda>0$ the energy functional $J: W_{0}^{1, p}(\Omega) \rightarrow \mathbb{R}$ by

$$
J(u)=\frac{1}{p} \int_{\Omega}(H(\nabla u))^{p} d x-\frac{\lambda}{2} \int_{\Omega} u^{2} d x, \forall u \in W_{0}^{1, p}(\Omega) .
$$

We establish some basic properties of $J$.

Proposition 1. For each $\lambda>0$, the functional $J$ is well-defined on $W_{0}^{1, p}(\Omega)$ and $J \in C^{1}\left(W_{0}^{1, p}(\Omega) \backslash\{0\}, \mathbb{R}\right)$ with the derivative given by

$$
\left\langle J^{\prime}(u), \varphi\right\rangle=\int_{\Omega}(H(\nabla u))^{p-2} \nabla H(\nabla u) \nabla \varphi d x-\lambda \int_{\Omega} u \varphi d x,
$$

for all $u \in W_{0}^{1, p}(\Omega) \backslash\{0\}$ and $\varphi \in W_{0}^{1, p}(\Omega)$. 
The proof of Proposition 1 follows from two results which can be found in Badiale and Serra [1, Theorem 2.6.4] and Willem [7, Proposition 1.12]. We recall their proofs for readers' convenience.

Lemma 3. Let $\Omega \subseteq \mathbb{R}^{N}(N \geq 3)$ be an open set. For $p>2$, define a functional $G: W_{0}^{1, p}(\Omega) \rightarrow \mathbb{R} b y$

$$
G(u)=\int_{\Omega}(H(\nabla u))^{p} d x .
$$

Then $G$ is differentiable in $W_{0}^{1, p}(\Omega) \backslash\{0\}$ and

$$
\left\langle G^{\prime}(u), v\right\rangle=p \int_{\Omega}(H(\nabla u))^{p-2} \nabla H(\nabla u) \nabla v d x
$$

for all $u \in W_{0}^{1, p}(\Omega) \backslash\{0\}$ and $v \in W_{0}^{1, p}(\Omega)$.

Proof. We consider the function $\varphi: \mathbb{R}^{N} \rightarrow \mathbb{R}$, defined by $\varphi(x)=H(x)^{p}$. It is a $C^{1}$-function and $\nabla \varphi(x)=p H(x)^{p-2} \nabla H(x)$, so that, for all $x \in \mathbb{R}^{N} \backslash\{0\}$ and $y \in \mathbb{R}^{N}$,

$$
\lim _{t \rightarrow 0} \frac{\varphi(x+t y)-\varphi(x)}{t}=p H(x)^{p-2} \nabla H(x) y .
$$

As a consequence for each $u \in W_{0}^{1, p}(\Omega) \backslash\{0\}$ and $\varphi \in W_{0}^{1, p}(\Omega)$ we have $\lim _{t \rightarrow 0} \frac{H(\nabla(u(x)+t v(x)))^{p}-(H(\nabla u(x)))^{p}}{t}=p H(\nabla u(x))^{p-2} \nabla H(\nabla u(x)) \nabla v(x)$, a.e. in $\Omega$. By the mean value theorem there exists $\theta \in \mathbb{R}$ such that $|\theta| \leq|t|$ and

$$
\begin{gathered}
\left|\frac{(H(\nabla(u+t v)))^{p}-(H(\nabla u))^{p}}{t}\right| \leq p\left|(H(\nabla(u+\theta v)))^{p-2} \nabla H(\nabla(u+\theta v)) \nabla v\right| \\
\leq C\left((H(\nabla u))^{p-1}|\nabla v|+|\nabla v|^{p}\right) \in L^{1}(\Omega) .
\end{gathered}
$$

By the dominated convergence theorem of Lebesgue we obtain

$$
\lim _{t \rightarrow 0} \int_{\Omega} \frac{(H(\nabla(u+t v)))^{p}-H(\nabla u)^{p}}{t} d x=p \int_{\Omega}(H(\nabla u))^{p-2} \nabla H(\nabla u) \nabla v d x,
$$

so that $G$ is Gâteaux differentiable and

$$
\left\langle G^{\prime}(u), v\right\rangle=p \int_{\Omega}(H(\nabla u))^{p-2} \nabla H(\nabla u) \nabla v d x .
$$

We have now to prove that $G^{\prime}: W_{0}^{1, p}(\Omega) \rightarrow\left[W^{1, p}(\Omega)\right]^{\prime}$ is continuous. To this aim we take a sequence $\left\{u_{k}\right\}_{k}$ in $W_{0}^{1, p}(\Omega)$ such that $u_{k} \rightarrow u$ in $W_{0}^{1, p}(\Omega)$. In particular we can assume, as usual, that up to subsequences, 
- $\nabla u_{k} \rightarrow \nabla u$ in $\left(L^{p}(\Omega)\right)^{N}$ as $k \rightarrow \infty$;

- $\nabla u_{k}(x) \rightarrow \nabla u(x)$ a.e. in $\Omega$ as $k \rightarrow \infty$;

- there exists $w \in L^{1}(\Omega)$ such that $\left|\nabla u_{k}(x)\right|^{p} \leq w(x)$ a.e. in $\Omega$ and for all $k \in \mathbb{N}$.

We have

$$
\begin{aligned}
\left\langle G^{\prime}(u)-G^{\prime}\left(u_{k}\right), v\right\rangle= & p \int_{\Omega}(H(\nabla u))^{p-2} \nabla H(\nabla u) \nabla v d x \\
& -p \int_{\Omega}\left(H\left(\nabla u_{k}\right)\right)^{p-2} \nabla H\left(\nabla u_{k}\right) \nabla v d x
\end{aligned}
$$

and by Hölder's inequality we get

$$
\begin{aligned}
& \left|\int_{\Omega}\left((H(\nabla u))^{p-2} \nabla H(\nabla u)-\left(H\left(\nabla u_{k}\right)\right)^{p-2} \nabla H\left(\nabla u_{k}\right)\right) \nabla v d x\right| \\
\leq & \left(\int_{\Omega}\left|\left(H\left(\nabla u_{k}\right)\right)^{p-2} \nabla H\left(\nabla u_{k}\right)-(H(\nabla u))^{p-2} \nabla H(\nabla u)\right|^{\frac{p}{p-1}} d x\right)^{\frac{p-1}{p}} \\
& \left(\int_{\Omega}|\nabla v|^{p} d x\right)^{\frac{1}{p}} \\
\leq & \left(\int_{\Omega}\left|\left(H\left(\nabla u_{k}\right)\right)^{p-2} \nabla H\left(\nabla u_{k}\right)-(H(\nabla u))^{p-2} \nabla H(\nabla u)\right|^{\frac{p}{p-1}} d x\right)^{\frac{p-1}{p}}\|v\|,
\end{aligned}
$$

so that

$$
\begin{aligned}
& \left\|G^{\prime}(u)-G^{\prime}\left(u_{k}\right)\right\|=\sup \left\{\left|\left\langle G^{\prime}(u)-G^{\prime}\left(u_{k}\right), v\right\rangle\right| ; v \in W^{1, p}(\Omega),\|v\|=1\right\} \\
& \leq\left(\int_{\Omega}\left|\left(H\left(\nabla u_{k}\right)\right)^{p-2} \nabla H\left(\nabla u_{k}\right)-(H(\nabla u))^{p-2} \nabla H(\nabla u)\right|^{\frac{p}{p-1}} d x\right)^{\frac{p-1}{p}} .
\end{aligned}
$$

Now we know that

$$
\left(H\left(\nabla u_{k}(x)\right)\right)^{p-2} \nabla H\left(\nabla u_{k}(x)\right) \rightarrow(H(\nabla u(x)))^{p-2} \nabla H(\nabla u(x))
$$

almost everywhere in $\Omega$, and that

$$
\begin{aligned}
&\left|\left(H\left(\nabla u_{k}\right)\right)^{p-2} \nabla H\left(\nabla u_{k}\right)-(H(\nabla u))^{p-2} \nabla H(\nabla u)\right|^{\frac{p}{p-1}} \\
& \leq C\left(\left(H\left(\nabla u_{k}\right)\right)^{p}+(H(\nabla u))^{p}\right) \\
& \leq C\left(w+(H(\nabla u))^{p}\right) \in L^{1}(\Omega) .
\end{aligned}
$$


By Lebesgue's dominated convergence theorem we obtain

$$
\int_{\Omega}\left|\left(H\left(\nabla u_{k}\right)\right)^{p-2} \nabla H\left(\nabla u_{k}\right)-(H(\nabla u))^{p-2} \nabla H(\nabla u)\right|^{\frac{p}{p-1}} d x \rightarrow 0,
$$

and hence $\left\|G^{\prime}(u)-G^{\prime}\left(u_{k}\right)\right\| \rightarrow 0$. This holds for a subsequence of the original sequence $\left\{u_{k}\right\}$, but, actually, we can obtain that $G^{\prime}$ is a continuous function, so that $G$ is differentiable with the derivative given by

$$
\left\langle G^{\prime}(u), v\right\rangle=p \int_{\Omega}(H(\nabla u))^{p-2} \nabla H(\nabla u) \nabla v d x .
$$

Lemma 4. Let $\Omega$ be an open subset of $\mathbb{R}^{N}$. The functional

$$
\mathcal{H}(u)=\int_{\Omega} u^{2} d x
$$

is of class $C^{1}\left(L^{2}(\Omega), \mathbb{R}\right)$ and

$$
\left\langle\mathcal{H}^{\prime}(u), h\right\rangle=2 \int_{\Omega} u h d x, \quad \forall u, h \in L^{2}(\Omega) .
$$

Proof. Existence of the Gâteaux derivative. Let $u, h \in L^{2}(\Omega)$. Given $x \in \Omega$ and $0<|t|<1$, by the mean value theorem, there exists $\lambda \in[0,1]$ such that

$$
\begin{gathered}
\left|\frac{|u(x)+t h(x)|^{2}-|u(x)|^{2}}{|t|}\right|=2|u(x)+\lambda t h(x)||h(x)| \\
\leq 2(|u(x)|+|h(x)|)|h(x)| .
\end{gathered}
$$

Hölder's inequality implies that

$$
(|u(x)|+|h(x)|)|h(x)| \in L^{1}(\Omega) .
$$

It follows then from Lebesgue's theorem that

$$
\left\langle\mathcal{H}^{\prime}(u), h\right\rangle=2 \int_{\Omega} u h d x .
$$

Continuity of the Gâteaux derivative. Let us define $f(u):=2 u$. Assume that $u_{n} \rightarrow u$ in $L^{2}(\Omega)$. Then $f\left(u_{n}\right) \rightarrow f(u)$ in $L^{2}$. We obtain, by Hölder's inequality,

$$
\left|\left\langle\mathcal{H}^{\prime}\left(u_{n}\right)-\mathcal{H}^{\prime}(u), h\right\rangle\right| \leq\left|f\left(u_{n}\right)-f(u)\right|_{L^{2}(\Omega)}|h|_{L^{2}(\Omega)},
$$

and so

$$
\left\|\mathcal{H}^{\prime}\left(u_{n}\right)-\mathcal{H}^{\prime}(u)\right\| \leq\left|f\left(u_{n}\right)-f(u)\right|_{L^{2}(\Omega)} \rightarrow 0, \text { as } n \rightarrow \infty
$$


Combining Lemmas 3 and 4 we infer that Proposition 1 holds true.

Proposition 2. If $J \in C^{1}\left(W_{0}^{1, p}(\Omega) \backslash\{0\}, \mathbb{R}\right)$ possesses a global minimum on $W_{0}^{1, p}(\Omega)$ achieved in $u_{0} \in W_{0}^{1, p}(\Omega) \backslash\{0\}$, then $u_{0}$ is a critical point of $J$.

Proof. Let $\varphi \in W_{0}^{1, p}(\Omega)$ be arbitrary but fixed. We define the function $f$ : $\mathbb{R} \rightarrow \mathbb{R}$ by

$$
f(\epsilon)=J\left(u_{0}+\epsilon \varphi\right)=\frac{1}{p} \int_{\Omega}\left(H\left(\nabla\left(u_{0}+\epsilon \varphi\right)\right)\right)^{p} d x-\frac{\lambda}{2} \int_{\Omega}\left(u_{0}+\epsilon \varphi\right)^{2} d x .
$$

The function $f$ has a minimum at $\epsilon=0$ and is differentiable with the derivative given by

$$
\begin{aligned}
f^{\prime}(\epsilon) & =\lim _{\epsilon \rightarrow 0} \frac{J\left(u_{0}+\epsilon \varphi\right)-J\left(u_{0}\right)}{\epsilon}=\left\langle J^{\prime}\left(u_{0}+\epsilon \varphi\right), \varphi\right\rangle \\
& =\int_{\Omega}\left(H\left(\nabla\left(u_{0}+\epsilon \varphi\right)\right)\right)^{p-2} \nabla H\left(\nabla\left(u_{0}+\epsilon \varphi\right)\right) \nabla \varphi d x-\lambda \int_{\Omega}\left(u_{0}+\epsilon \varphi\right) \varphi d x .
\end{aligned}
$$

Therefore

$$
0=f^{\prime}(0)=\int_{\Omega}\left(H\left(\nabla u_{0}\right)\right)^{p-2} \nabla H\left(\nabla u_{0}\right) \nabla \varphi d x-\lambda \int_{\Omega} u_{0} \varphi d x=\left\langle J^{\prime}\left(u_{0}\right), \varphi\right\rangle,
$$

which implies that $u_{0}$ is a critical point for $J$.

Lemma 5. The functional $J$ is coercive (i.e. $\lim _{\|u\|_{W_{0}^{1, p}(\Omega)} \rightarrow \infty} J(u)=\infty$ ) on $W_{0}^{1, p}(\Omega)$.

Proof. We know that

$$
\begin{gathered}
J(u)=\frac{1}{p} \int_{\Omega}(H(\nabla u))^{p} d x-\frac{\lambda}{2} \int_{\Omega} u^{2} d x \\
=\frac{1}{p} \int_{\Omega}(H(\nabla u))^{p} d x-\frac{\lambda}{2}\|u\|_{L^{2}(\Omega)}^{2} .
\end{gathered}
$$

Since any two norms are equivalent on $\mathbb{R}^{N}$ we infer that for $H$ defined as above there exists a positive constant $k$ such that

$$
\int_{\Omega}(H(\nabla u))^{p} d x \geq k \int_{\Omega}|\nabla u|^{p} d x=k\|u\|_{W_{0}^{1, p}}^{p} .
$$

We recall here that for every $2<p<\infty, W_{0}^{1, p}(\Omega)$ is continuously embedded in $L^{2}(\Omega)$ (see, e.g. [2, Corollary IX.14] or [3]), i.e. there exists $c>0$ such that $\|u\|_{L^{2}(\Omega)} \leq c\|u\|_{W_{0}^{1, p}(\Omega)}$. Taking into account the above facts, we obtain

$$
J(u) \geq \frac{k}{p}\|u\|_{W_{0}^{1, p}(\Omega)}^{p}-\frac{\lambda c^{2}}{2}\|u\|_{W_{0}^{1, p}(\Omega)}^{2} .
$$


From this inequality and taking into account that $p>2$, we deduce that $J$ is coercive.

Lemma 6. The functional $J$ is weakly lower semi-continuous (i.e. for every sequence $\left\{u_{n}\right\}_{n \in \mathbb{N}} \subset W_{0}^{1, p}(\Omega)$ converging weakly to $u \in W_{0}^{1, p}(\Omega)$, we have $\left.J(u) \leq \liminf _{n \rightarrow \infty} J\left(u_{n}\right)\right)$ on $W_{0}^{1, p}(\Omega)$.

Proof. We take $\left\{u_{n}\right\}_{n \in \mathbb{N}} \subset W_{0}^{1, p}(\Omega)$ converging weakly to $u \in W_{0}^{1, p}(\Omega)$. Since $H$ is convex by $[2$, Proposition III.5 (iii)] we have

$$
\frac{1}{p} \int_{\Omega}(H(\nabla u))^{p} d x \leq \liminf _{n \rightarrow \infty} \frac{1}{p} \int_{\Omega}\left(H\left(\nabla u_{n}\right)\right)^{p} d x .
$$

Since $W_{0}^{1, p}(\Omega)$ is compactly embedded in $L^{2}(\Omega)$ (see [2, Theorem IX.16] or $[3])$, we obtain that $\left\{u_{n}\right\}$ converges strongly to $u$ in $L^{2}(\Omega)$. It follows that

$$
\frac{\lambda}{2} \int_{\Omega} u_{n}^{2} d x \rightarrow \frac{\lambda}{2} \int_{\Omega} u^{2} d x
$$

From relations (6) and (7), we conclude that

$$
J(u) \leq \liminf _{n \rightarrow \infty} J\left(u_{n}\right) .
$$

Lemma 7. There exists $\theta \in W_{0}^{1, p}(\Omega) \backslash\{0\}$ such that $J(\theta)<0$.

Proof. Let $\theta \in W_{0}^{1, p}(\Omega) \backslash\{0\}$ be fixed. For each $t>0$ we have

$$
J(t \theta)=\frac{t^{p}}{p} \int_{\Omega}(H(\nabla \theta))^{p} d x-\frac{\lambda t^{2}}{2} \int_{\Omega} \theta^{2} d x .
$$

As $p>2$, for any $t \in\left(0,\left[\frac{\lambda p \int_{\Omega} \theta^{2} d x}{2 \int_{\Omega}(H(\nabla \theta))^{p} d x}\right]^{\frac{1}{p-2}}\right)$ we have $J(t \theta)<0$.

Proof of Theorem 1 (When $p \in(2, \infty)$ ). By Lemmas 5 and 6 we deduce that $J$ is coercive and weakly lower semi-continuous on $W_{0}^{1, p}(\Omega)$. Then Theorem 3 implies that there exists a point $u_{\lambda} \in W_{0}^{1, p}(\Omega)$ such that $J\left(u_{\lambda}\right)=$ $\min _{v \in W_{0}^{1, p}(\Omega)} J(v)$. Since $J\left(u_{\lambda}\right)=J\left(\left|u_{\lambda}\right|\right)$ we may assume that $u_{\lambda} \geq 0$ in $\Omega$.

By Lemma 7 we obtain that there exists $\theta \in W_{0}^{1, p}(\Omega)$ such that $J(\theta)<0$. By these remarks we can say that $J\left(u_{\lambda}\right)<0$, which implies $u_{\lambda} \neq 0$. As $J \in$ $C^{1}\left(W_{0}^{1, p}(\Omega) \backslash\{0\}, \mathbb{R}\right)$, by Proposition $2,\left\langle J^{\prime}\left(u_{\lambda}\right), v\right\rangle=0$, for any $v \in W_{0}^{1, p}(\Omega)$ and thus $u_{\lambda}$ is a nontrivial weak solution of problem (1). 
Finally, note that the Direct Method in Calculus of Variations can not be applied in order to prove Theorem 1 when $N \geq 3$ and $p \in\left(\frac{2 N}{N+2}, 2\right)$ since in this case we can not establish the coercivity of the functional $J$ which is an essential requirement in Theorem 3.

Conclusion. In this paper we emphasized two different situations regarding the nature of the spectrum of the eigenvalue problem (1). More precisely, first, we recalled the well-known fact that if $H$ is the euclidian norm on $\mathbb{R}^{N}$ and $p=2$ then the spectrum of problem (1) is discrete and, next, we showed that if $H$ is a general Finsler norm and $p \in\left(\frac{2 N}{N+2}, \infty\right) \backslash\{2\}$ then the spectrum of problem (1) is continuous. Moreover, regarding the corresponding eigenfunctions we pointed out the fact that they change sign when $p=2$ and we showed that they are one-signed when $p \in\left(\frac{2 N}{N+2}, \infty\right) \backslash\{2\}$. In particular, we extended some known results from Ôtani [5] to the case of Finsler $p$-Laplacian.

Acknowledgements. The author would like to thank the anonymous referee for the careful reading of the original manuscript and for a number of relevant comments that have lead to improvements in the exposition.

\section{References}

[1] M. Badiale and E. Serra: Semilinear elliptic equations for begginers, Springer, 2011.

[2] H. Brezis: Analyse fonctionnelle: théorie, méthodes et applications, Masson, Paris, 1992.

[3] H. Brezis: Functional Analysis, Sobolev Spaces and Partial Differential Equations, Universitext, Springer, New York, 2011, xiv+599 pp.

[4] V. Ferone and B. Kawohl: Remarks on a Finsler-Laplacian, Proc. Amer. Math. Soc. 137 (2009) 247-253.

[5] M. Ôtani: Existence and nonexistence of nontrivial solutions of some nonlinear degenerate elliptic equations, J. Funct. Anal. 76 (1988) 140-159.

[6] M. Struwe: Variational Methods: Applications to Nonlinear Partial Differential Equations and Hamiltonian Systems, Springer, Heidelberg, 1996.

[7] M. Willem: Minimax theorems, Birkhäuser, Boston, 1996.

[8] M. Willem: Analyse fonctionnelle élémentaire, Cassini, Paris, 2003. 
EIGENVALUES FOR $P$-LAPLACIAN WITH ZERO DIRICHLET BOUNDARY CONDITION

[9] M. Willem: Functional Analysis. Fundamentals and Applications, Birkhäuser/Springer, New York, 2013. xiv+213 pp.

Maria FĂRCĂŞEANU,

Department of Mathematics,

University of Craiova,

200585 Craiova, Romania.

Email: farcaseanu.maria@yahoo.com 